\title{
Genome expansion of Arabis alpina linked with retrotransposition and reduced symmetric DNA methylation
}

\author{
Eva-Maria Willing , Vimal Rawat , Terezie Mandáková , Florian Maumus, Geo Velikkakam James , \\ Karl J.V. Nordström , Claude Becker, Norman Warthmann , Claudia Chica , Bogna Szarzynska , \\ Matthias Zytnicki , Maria C. Albani , Christiane Kiefer, Sara Bergonzi , Loren Castaings , Julieta \\ L. Mateos , Markus C. Berns, Nora Bujdoso , Thomas Piofczyk, Laura de Lorenzo , \\ Cristina Barrero-Sicilia , Isabel Mateos , Mathieu Piednoël , Jörg Hagmann , Romy Chen-Min-Tao , \\ Raquel Iglesias-Fernández, Stephan C. Schuster, Carlos Alonso-Blanco, François Roudier', \\ Pilar Carbonero, Javier Paz-Ares, Seth J. Davis , Ales Pecinka, Hadi Quesneville, Vincent Colot , \\ Martin A. Lysak, Detlef Weigel , George Coupland and Korbinian Schneeberger
}

\begin{abstract}
Despite evolutionary conserved mechanisms to silence transposable element activity, there are drastic differences in the abundance of transposable elements even among closely related plant species. We conducted a de novo assembly for the $375 \mathrm{Mb}$ genome of the perennial model plant, Arabis alpina. Analysing this genome revealed long-lasting and recent transposable element activity predominately driven by Gypsy long terminal repeat retrotransposons, which extended the lowrecombining pericentromeres and transformed large formerly euchromatic regions into repeat-rich pericentromeric regions. This reduced capacity for long terminal repeat retrotransposon silencing and removal in A. alpina co-occurs with unexpectedly low levels of DNA methylation. Most remarkably, the striking reduction of symmetrical CG and CHG methylation suggests weakened DNA methylation maintenance in A. alpina compared with Arabidopsis thaliana. Phylogenetic analyses indicate a highly dynamic evolution of some components of methylation maintenance machinery that might be related to the unique methylation in A. alpina.
\end{abstract}

Whole-genome sequences of members of the Brassicaceae family ${ }^{1-10}$ (including the plant model $A$. thaliana) are greatly expanding the scope for comparative genomics among closely related plant species (for example refs 5,8 ). Despite their close phylogenetic relationship, Brassicaceae species have repeatedly evolved many differences in important life history traits, including the capacity for self-fertilization ${ }^{11}$, senescence ${ }^{12}$ and annual or perennial flowering behavior ${ }^{13}$.

The reference accession of the perennial A. alpina Pajares was collected in the Cordillera Cantábrica mountains of Spain and was self-fertilized for six generations by single-seed descent ${ }^{13}$. We generated a high-quality $309 \mathrm{Mb}$ genome assembly from a mixture of 454 Illumina and Sanger BAC end sequences with scaffolds with an N50 of $788 \mathrm{~kb}$ and L50 of $160 \mathrm{~kb}$ (Supplementary Fig. 1, Supplementary Tables 1 and 2 and Supplementary Note). By integrating comparative chromosome painting, we arranged more than $85 \%$ of the large scaffolds ( $>50 \mathrm{~kb}$ ) into eight pseudomolecules representing the eight chromosomes of $A$. alpina
(Supplementary Figs 2-4 and Supplementary Note). During a manual annotation jamboree the structural annotation of 514 selected genes was curated from a total of 30,729 genes and 278,110 repetitive elements (Supplementary Tables 3 and 4 and Supplementary Note). To resolve the phylogenetic placement of A. alpina, we calculated a whole genome-based phylogenetic consensus network for 10 Brassicaceae species with available reference assemblies (Fig. 1a). In addition to its topology, neutral variation and chromosome rearrangements specific to the karyotype of A. alpina argue for A. alpina being a member of a separate lineage that diverged from the Arabidopsis lineage around $27 \pm 16 \mathrm{Ma}$ (Fig. 1b, Supplementary Figs 5-7 and Supplementary Note).

Within current annotations of Brassicaceae genomes, genes and introns account for $58-81 \mathrm{Mb}$, whereas transposable element content is much more variable and accounts for up to $148 \mathrm{Mb}$ in A. alpina (Fig. 1c). By far the most abundant TE superfamily in A. alpina is the long terminal repeat (LTR) retrotransposons superfamily Ty3/Gypsy (or Gypsies) (Supplementary Table 5). An increasing number of plant genomes have pointed to recent bursts of LTR retrotransposon transposition as a common phenomenon ${ }^{2,14}$. A hallmark of such recent transpositions is large amounts of young copies $^{9}$ ( $>95 \%$ sequence similarity). However, in A. alpina we found a large fraction of middle-aged transposable elements (85-95\% sequence similarity $)^{15}$. The reduced amount of very young elements most likely indicates a recent reduction of Gypsy element activity as only small parts of this result from inefficiencies in short read assembly. Rather large parts of the non-assembled sequence relates to simple sequence repeats (Supplementary Figs 8 and 9 and Supplementary Note). Intriguingly, only the Gypsies contribute to the high number of middle-aged transposable elements in A. alpina (Fig. 2a and Supplementary Fig. 10). This suggests that A. alpina Gypsy elements proliferated over an extended period of time and that a large amount of these elements was retained and removed at only slow rates ${ }^{16}$.

To analyse the degree to which these TEs may be epigenetically silenced, we assayed four distinct chromatin marks in $A$. alpina, A. thaliana and A. lyrata, including histone modifications 

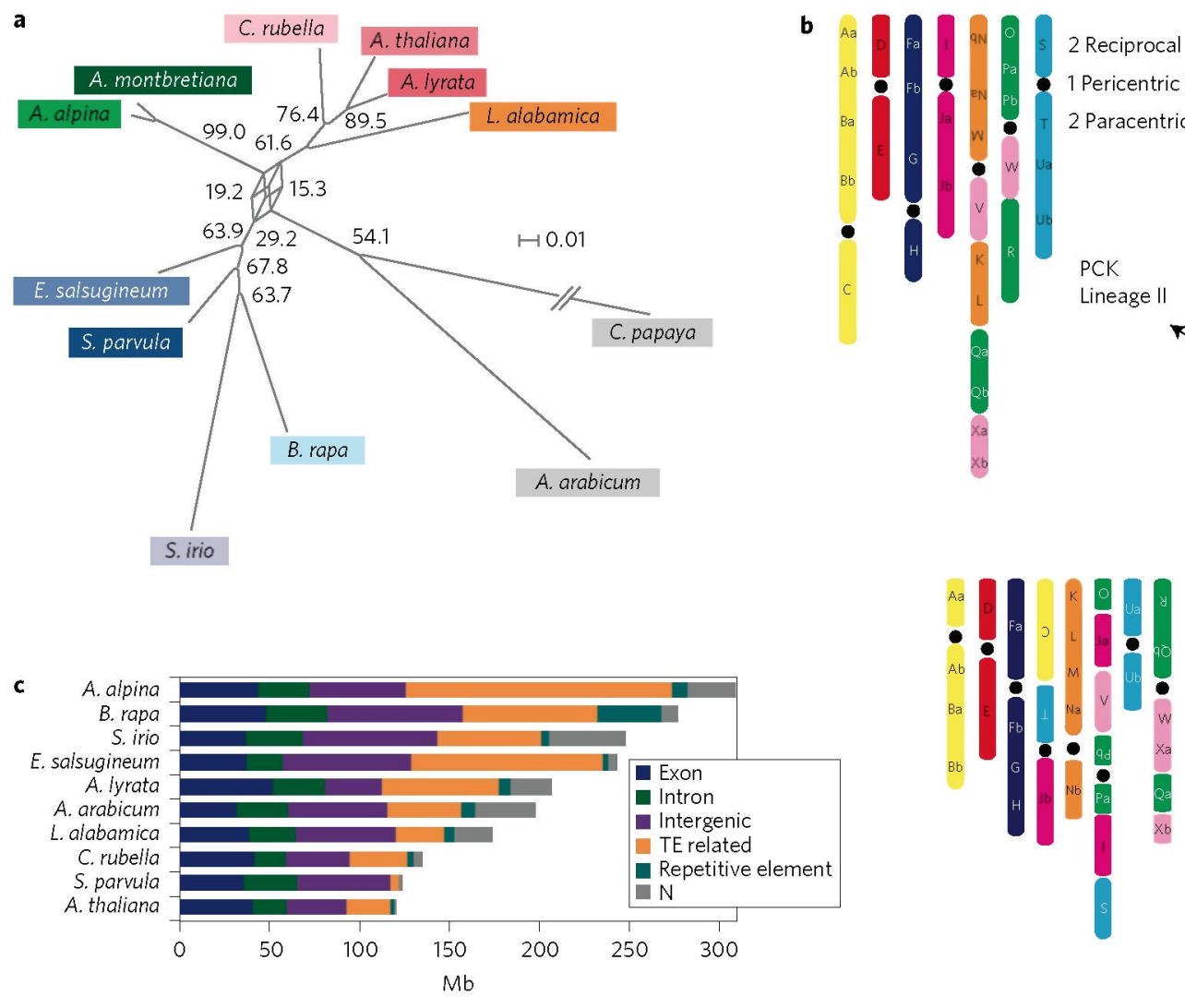

Figure 1 | Phylogenetic reconstruction and karyotype evolution support a distinct phylogenetic placement of $A$. alpina. a, Consensus network of the Brassicaceae phylogeny based on 1,787 single-copy clusters of orthologous genes. Its topology did not unambiguously place the Arabis species with Lineage II as proposed earlier. (Lineage I species (red); Lineage II species (blue); Arabis species (green)). b. Karyotype evolution at the base of Brassicaceae evolution. Reconstruction of the chromosome evolution from the Ancestral Crucifer Karyotype (ACK) to the A. alpina karyotype (KAA) suggested nine chromosomal rearrangements, which are different from the rearrangements that occurred in the evolution from the ACK to the Proto-Calepineae Karyotype (PCK), which is ancestral to Lineage II. c, Brassicaceae genome compositions. TEs, transposable elements

H3K4me3, H3K27me3 and H3K27mel assayed by ChIP-seq, and DNA methylation assayed by immunoprecipitation of methylated DNA coupled with high-throughput sequencing ${ }^{17}$ (Supplementary Note). H3K27me1 and DNA methylation mark epigenetically silenced TEs, whereas $\mathrm{H} 3 \mathrm{~K} 27 \mathrm{me} 3$ is a repressive mark specifically associated with genes and $\mathrm{H} 3 \mathrm{~K} 4 \mathrm{me} 3$ is associated with regions that are actively transcribed ${ }^{18}$. As in $A$. thaliana ${ }^{18}$, DNA methylation and H3K27me1 modification were mostly associated with TEs in A. alpina and A. lyrata, whereas $\mathrm{H} 3 \mathrm{~K} 4 \mathrm{me} 3$ and $\mathrm{H} 3 \mathrm{~K} 27 \mathrm{me} 3$ were preferentially associated with genes (Fig. $2 \mathrm{~b}$ and Supplementary Fig. 11). In A. alpina, however, we found a three times larger proportion of TEs marked with H3K4me3 as in the other two species. Gypsies showed by far the largest fraction of elements marked with H3K4me3, whereas all other superfamilies did not show such a pronounced increase (Fig. 2b and Supplementary Figs 10, 11).

Even though Gypsies within genes were more likely to be marked with $\mathrm{H} 3 \mathrm{~K} 4 \mathrm{me} 3$ and were consistently older in all three species (Fig. 2c), A. alpina showed only a slightly increased fraction (7.8\%) of Gypsies in genes compared with A. thaliana $(5.7 \%)$ and much less than $A$. lyrata (15.1\%), implying that elevated levels of H3K4me3 marking among Gypsies were not dominated by epigenetic states of genes. Moreover, H3K4me3-marked Gypsy elements outside of genes were drastically younger than those without this mark in A. thaliana and A. lyrata but not in A. alpina, suggesting that many of these elements might have retained the ability to be transcribed over time. In fact, when analysing the $1.5 \%$ of the RNA-sequencing (seq) reads that were not assigned to genes ${ }^{9,19}$, we found that the Gypsy superfamily was more expressed than any of the other superfamilies in A. alpina, in contrast to A. thaliana in which Copia showed the highest fraction of RNA-seq reads (Fig. 2d, Supplementary Fig. 12 and Supplementary Note). Moreover, transposable elements with the H3K4me3 mark were significantly enriched for expressed TEs compared with TEs without this mark across all large superfamilies, even though this effect was less pronounced for Gypsies (Fig. 2e).

Two Gypsy families, ATGPI and ATLANTYS2, accounted for more than a fifth of all Gypsy elements in A. alpina (Fig. $2 \mathrm{f}$ and Supplementary Note). These two families showed an even more drastic increase in elements marked with $\mathrm{H} 3 \mathrm{~K} 4 \mathrm{me} 3$, which was not apparent in any of the other A. alpina TE families, and together with their increased copy number and age distribution this suggests that the observed burst of transposition was mostly driven by this small group of TEs.

In A. alpina, A. thaliana and A. lyrata, TE density increases towards the centromeres ${ }^{1,2}$ (Fig. 3a and Supplementary Figs 13 and 14). Typically these repeat-rich regions overlap with heterochromatic pericentromeres. Here we defined pericentromeres as regions with high amounts of $H 3 K 27 \mathrm{mel}$ surrounding the centromeres (Supplementary Table 6 and Supplementary Note). Pericentromeres in A. alpina were drastically larger (average length: $14.9 \mathrm{Mb}$ in $A$. alpina, $3.9 \mathrm{Mb}$ in $A$. thalian ${ }^{1}, 10.3 \mathrm{Mb}$ in A. lyrata $^{2}$ ) and included many more genes than the other two species (Fig. 3b). Gypsy elements are significantly enriched among the repeats in pericentromeres $(U$-test, $P$-value $<2 \mathrm{e}-16)$ and account for many of the size differences of pericentromeres (Fig. $3 \mathrm{c}$ ). In $A$. thaliana and A. lyrata $\mathrm{H} 3 \mathrm{~K} 4 \mathrm{me} 3$ markings are 

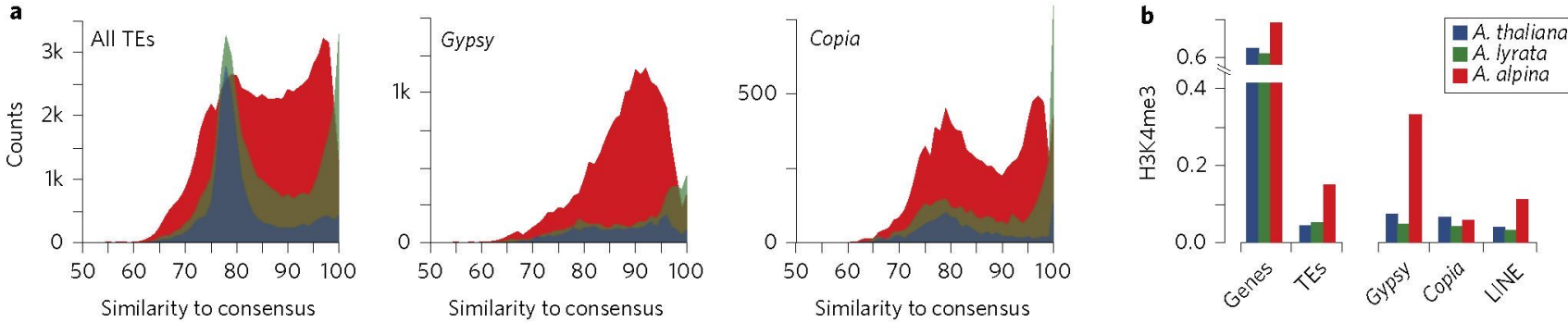

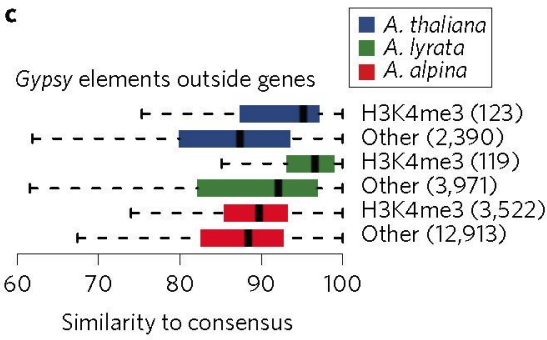

Gypsy elements inside genes

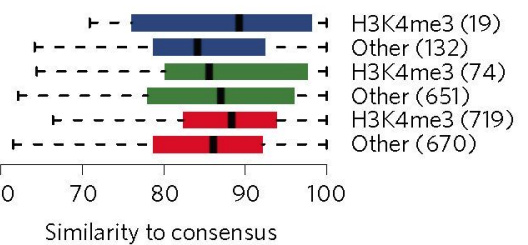

d
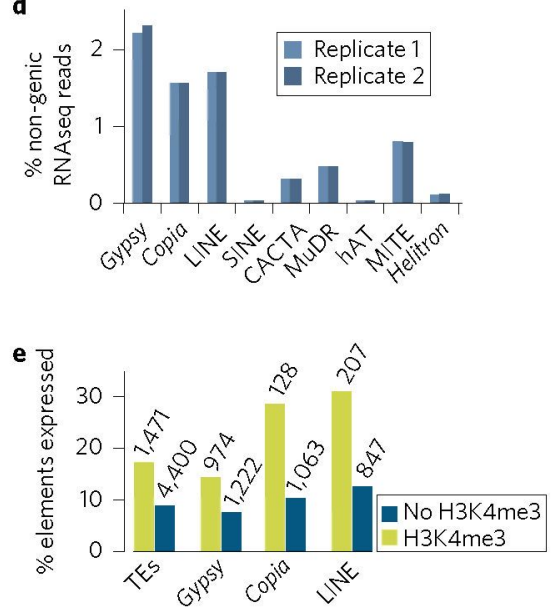

f

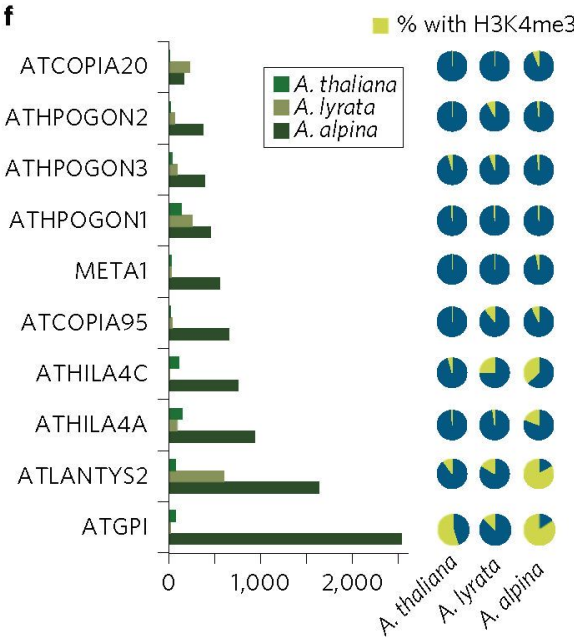

Figure 2 | Genome size variation and differences in transposable element content. a, transposable element age spectra based on similarity between transposable element copies and consensus sequence. A. alpina shows a unique increase in middle-aged Gypsy elements. $\mathbf{b}$, Fraction of genes and

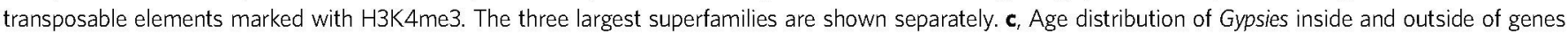
separated by their different $\mathrm{H} 3 \mathrm{~K} 4 \mathrm{me} 3$ markings. d, Expression of individual transposable element superfamilies in A. alpina. e, Fraction of expressed transposable elements with and without H3K4me3 markings. $\mathbf{f}$, Size of the ten largest transposable element families in A. alpina along with their family-wide fraction of $\mathrm{H} 3 \mathrm{~K} 4 \mathrm{me} 3$ marks within all three species.

strongly correlated with gene density throughout the chromosomes. In A. alpina, however, this correlation was weak and even entirely missing in pericentromeres, where $\mathrm{H} 3 \mathrm{~K} 4 \mathrm{me} 3$ was slightly correlated with Gypsy element density instead, suggesting that Gypsies are epigenetically active even in the heterochromatic pericentromere in A. alpina (Fig. 3a and Supplementary Figs 15-17).

Genome size differences between Brassicaceae species have previously been attributed to pericentromere expansion ${ }^{6,20}$, but the causes and functional consequences have remained unclear. Centromeres in many species suppress crossover recombination during meiosis, a phenomenon that usually extends into heterochromatic regions near the centromere ${ }^{21}$. Crossover frequencies along seven investigated chromosomes of A. alpina revealed for each chromosome a region with suppressed crossovers (Supplementary Tables 7,8 and Supplementary Note). These regions co-localize with large parts of the pericentromeres implying that the extent of non-recombining DNA in A. alpina is greatly increased compared with $A$. thaliana and $A$. lyrata.

Earlier analyses reported differences in gene content in pericentromeres of Brassicaceae ${ }^{20}$, but were complicated by the lack of whole-genome sequences. Reconstruction of ancestral chromosomal rearrangements of $A$. alpina revealed a single homologous paleocentromere (chromosome 2) with A. lyrata ${ }^{2}$ (Supplementary Figs 2 and 3). The assembly of the long arm of chromosome 2 shows a clear transition between gene- and repeat-rich regions in both species (Fig. 3d). Near the transition zone, there are 207 single-copy orthologues that reside in the repeat-dense regions in A. alpina, but outside the pericentromere in gene-rich regions in A. lyrata. Comparing two sparse genetic maps of these species suggested that the repeat-rich region in A. alpina shows more strongly suppressed recombination than the orthologous regions in A. lyrata (Fig. $3 \mathrm{~d}$ and Supplementary Note) ${ }^{22}$. This implies that upon expansion of the repeat-rich pericentromeric regions in A. alpina, genes in formerly gene-rich regions became incorporated into the pericentromere, with the consequence that large clusters of genes experience very little meiotic recombination in $A$. alpina. Although we cannot fully exclude the possibility of accelerated loss of transposable elements and pericentromere shrinkage in A. lyrata, we found no evidence for large numbers of solo LTRs that would indicate continuing loss through unequal homologous deletions in this particular genomic region (Fig. 3d and Supplementary Note).

Increased transposable element activity and gain of $\mathrm{H} 3 \mathrm{~K} 4 \mathrm{me} 3$ has been linked to reduction in DNA methylation at transposable elements in $A$. thalian ${ }^{23}$. To further examine DNA methylation in $A$. alpina, we performed whole-genome bisulphite sequencing ${ }^{24}$ using leaf material and compared it with analogous data previously generated for A. thaliana ${ }^{25}$ (Supplementary Note). Though this revealed similar amounts of methylated cytosines in A. alpina (19\%) and $A$. thaliana (16\%) ${ }^{25}$ and similar methylation profiles along genes and transposable elements in both species ${ }^{24}$ (Supplementary Fig. 18), the position-wise frequency of CG methylation was strikingly different. Whereas most methylated CGs showed $80-100 \%$ methylation in A. thaliana, these levels tended to be much lower in A. alpina irrespective of sequence annotation (Fig. 4a,b and Supplementary Fig. 19). In contrast, the distribution of methylation levels at CHG sites was only slightly shifted towards lower values in A. alpina, and was very similar for $\mathrm{CHH}$ sites. In A. thaliana, CG and CHG methylation typically occurs on both Cs of the opposite strands of these palindromes, 
A. thaliano chromosome
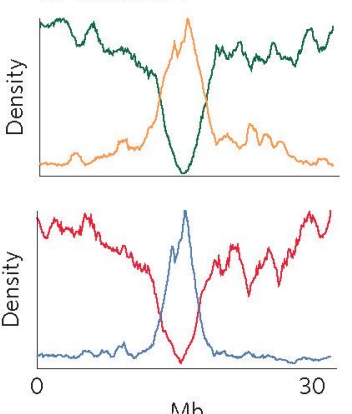

$\mathrm{Mb}$

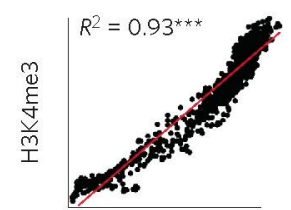

Gene density
A. lyrata

chromosome 2 chromosome 2
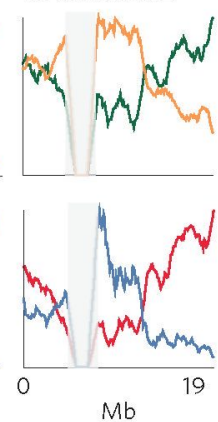

Mb

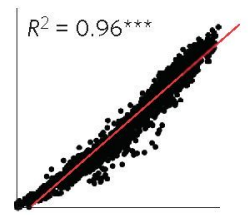

Gene density
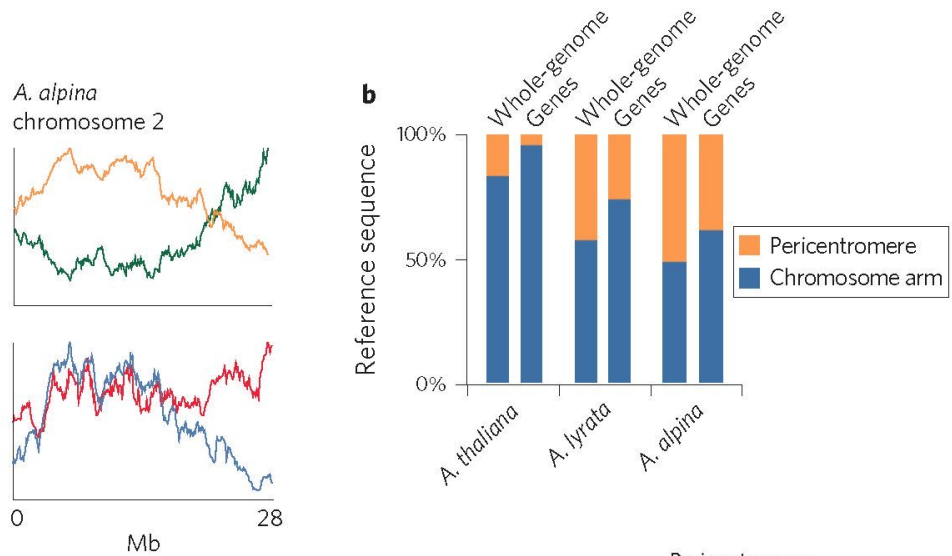

$c$
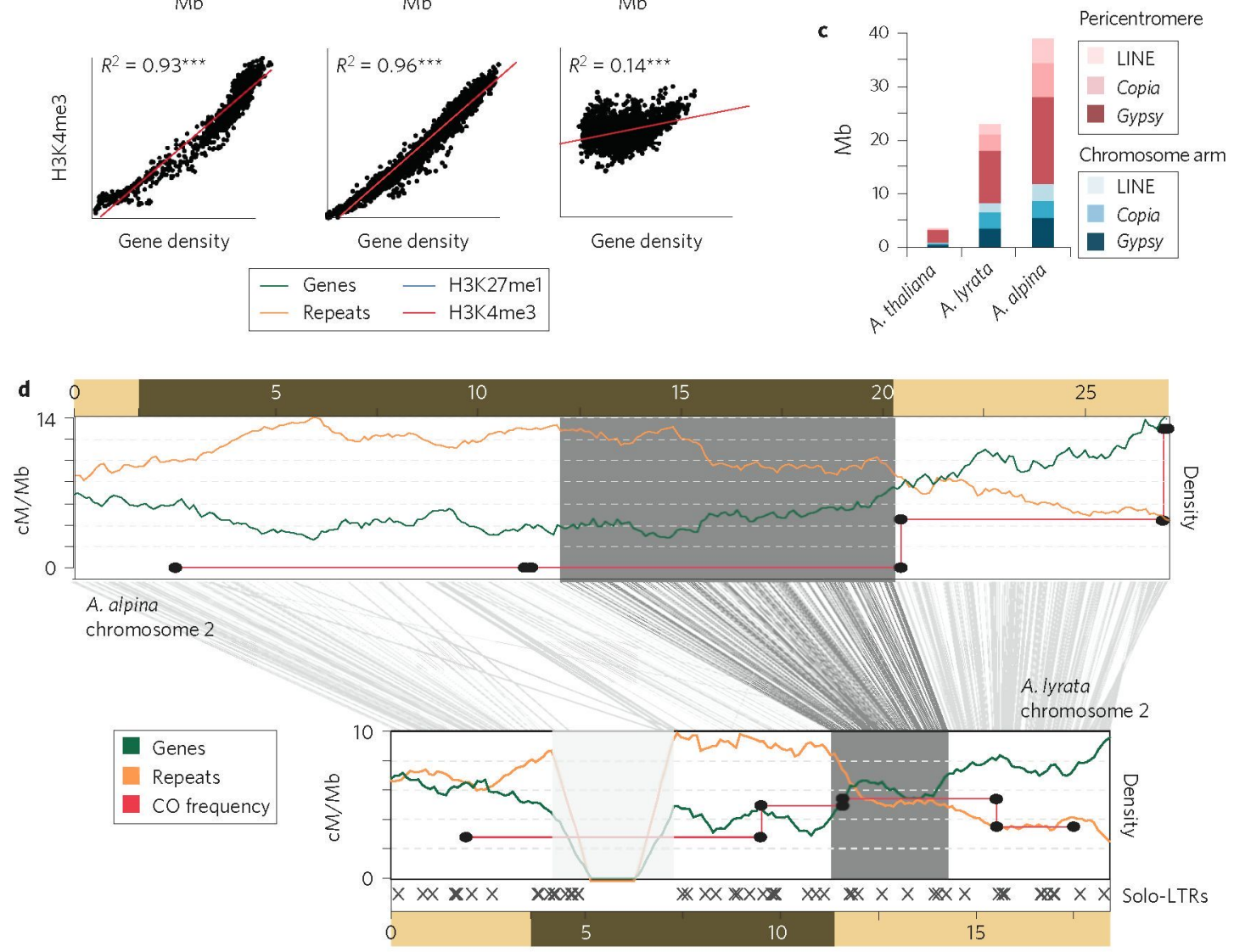

Figure 3 | Differences in the distribution of genes, transposable elements and chromatin marks between $A$. thaliana, $A$. lyrata and A. alpina. a, Gene, transposable element and histone mark density, along orthologous chromosomes (missing sequence marked in grey). $\mathbf{b}$, Genomic fraction and gene space in chromosome arms and pericentromeres. $\mathbf{c}$. Genome coverage of the three largest transposable element superfamilies. $\mathbf{d}$, Comparison of A. alpina and A. Jyrata chromosome 2 sharing the same ancestral centromere. Grey lines connect single-gene orthologues. Orthologues that reside in pericentromeric regions in A. alpina, but are outside these regions in A. lyrata, are indicated by dark grey lines. Locations of solo-LTRs indicated by grey crosses. (Gene and transposable element densities as in $\mathbf{a}$, crossover frequency (red), pericentromeres (dark brown)).

indicative of methylation copying via the maintenance machinery during replication ${ }^{24,26}$. Surprisingly, the two strands are essentially uncorrelated in their methylation levels at CG sites, and much more weakly correlated at $\mathrm{CHG}$ sites in A. alpina throughout the entire genome, suggesting that methylation maintenance is much less pervasive (Fig. 4c and Supplementary Fig. 20).

Given these fundamental differences, we suspected that the DNA methylation maintenance machinery might function differently in A. alpina. To explore this possibility, we examined the Brassicaceae genomes for intact homologues of the five major gene families involved in DNA methylation ${ }^{26}$ (Fig. $4 \mathrm{~d}$ ). At least one homologue of each family was found and showed expression in A. alpina (Supplementary Table 9). The phylogenies of DDM1, required for $C G$ methylation maintenance, CMT3, required for CHG methylation maintenance and DRM2, involved in de novo methylation in all contexts, broadly recapitulated the family phylogeny (Fig. $4 \mathrm{e}-\mathrm{g}$ ). In contrast, the homologues of MET1 and VIM1, which in addition to $D D M 1$ are essential for CG methylation maintenance in $A$. thaliana ${ }^{26}$, clustered in a species- and lineage-specific manner (Fig. 4h,i). This implies that all species outside the A rabidopsis lineage lacked clear orthologues for METI and VIMI genes, which was also apparent from the lack of synteny of these genes with any of their homologues outside this lineage. Moreover, the ratio of mutation rates at nonsynonymous $(N)$ and 

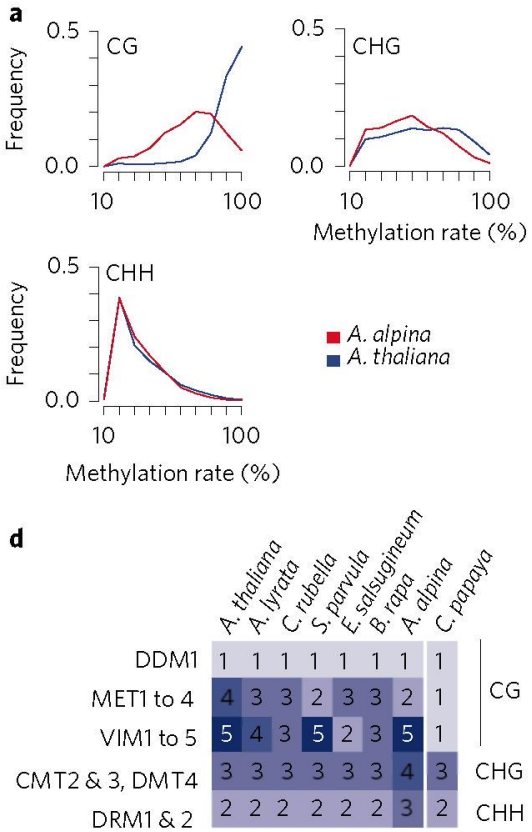

j

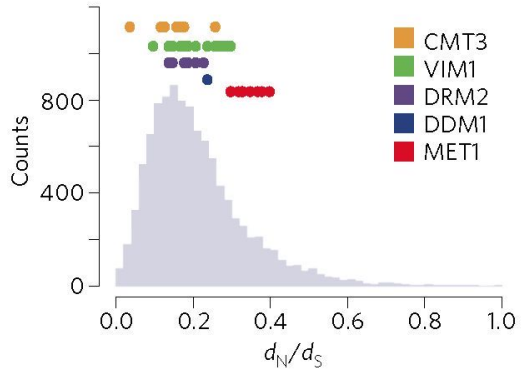

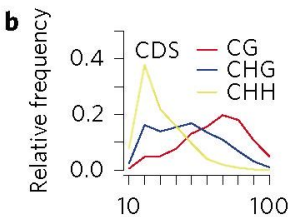

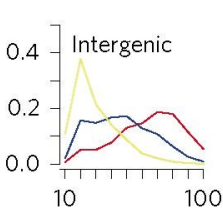

- A. alpina - A. thaliana

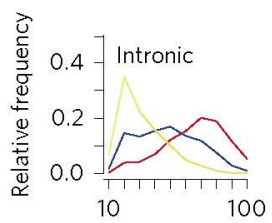

Methylation rate (\%)

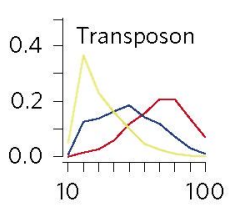

Methylation rate (\%)
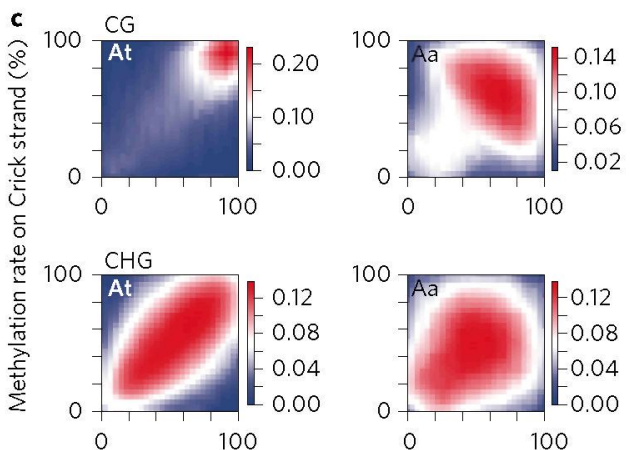

Methylation rate on Watson strand (\%)

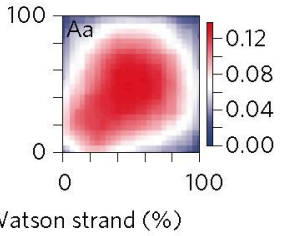

h

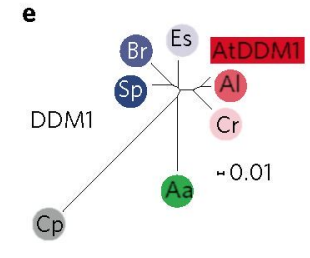

g
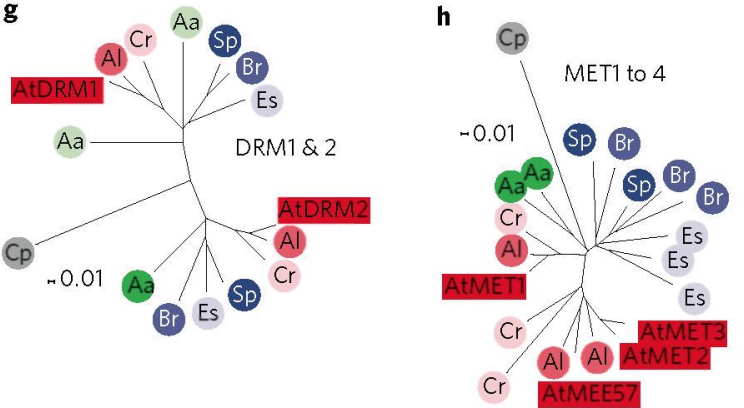
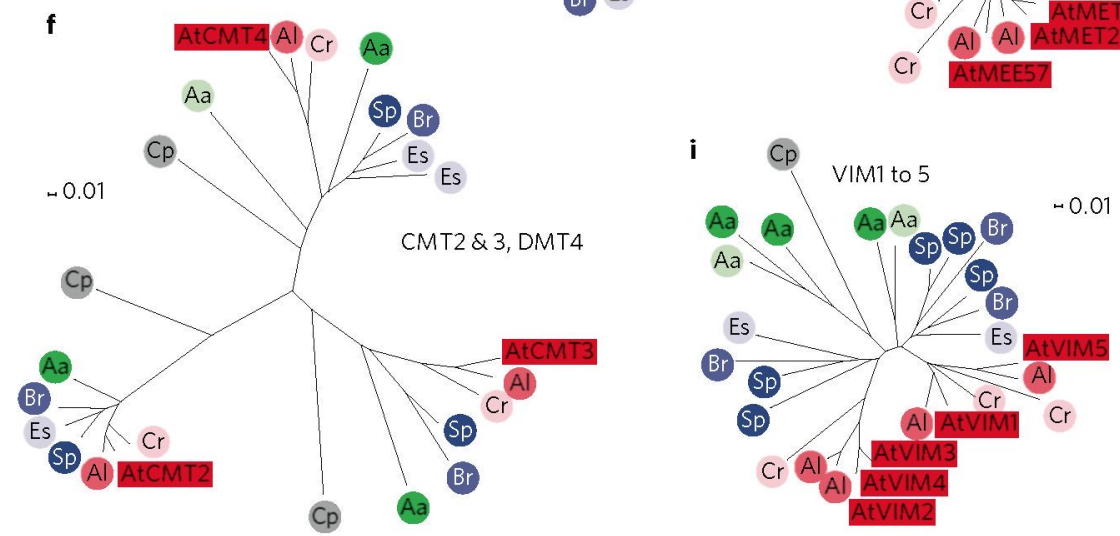

Figure 4 | Species-specific differences in DNA methylation. a, Position-wise DNA methylation frequencies. b, DNA methylation frequencies in A. alpina separated by genomic regions. c, Correlation of methylation frequency on Watson and Crick strand at symmetrical CG and $\mathrm{CHG}$ sites (Aa, $\mathrm{A}$. alpina; At, A. thaliana). d, Gene family sizes of DNA methylation genes. e-i, Gene family phylogenies (Aa, A. alpina; Al, A. lyrata, At, A. thaliana; Br, B. rapa; Cp, C. papaya, Cr, C. rubella; Es, E. salsugineum, Sp, S. parvula; Lineage I species (red); Lineage II species (blue); A. alpina (green)). $\mathbf{j}_{\text {, }} d_{N} / d_{S}$ values for orthologous genes pairs between $A$. alpina and A. thaliana (light blue) and $d_{N} / d_{S}$ values of each methylation gene family (coloured dots).

synonymous (S) sites $d_{\mathrm{N}} / d_{\mathrm{S}}$ calculated for each gene family revealed values highly similar to a genome-wide background distribution, except for MET1 family members with consistently enriched values, suggesting that less purifying selection pressure acts on MET1 (Fig. 4j).

Although MET1 and VIM1 homologues are present in A. alpina, it remains possible that the lineage-specific evolution of these genes might relate to the differences in CG methylation maintenance, as homologues of the main methylation genes are present in other species with strong differences in DNA methylation ${ }^{27}$. However, more complex changes in other methylation pathways might need to be considered to reveal the basis of DNA methylation differences between A. alpina and A. thaliana. As the absence of symmetrical CG methylation levels did not correlate with an overall lower amount of at least partially methylated cytosines, de novo DNA methylation probably compensates for the lack of DNA methylation maintenance ${ }^{28}$ underlining the high importance of de novo DNA methylation in A. alpina.

Even though co-occurrence of expanded transposable element content and DNA methylation maintenance deficiency in A. alpina does not necessarily imply a causal relationship, it nevertheless remains an attractive possibility that apparent methylation deficiency might have contributed to the elevated numbers of Gypsy elements, possibly due to reduced silencing of specific transposable element families, as was shown for DNA methylation maintenance deficient mutants in A. thaliana ${ }^{29,30}$.

\section{Data availability}

All data from this study have been deposited at the NCBI Sequence Read Archive (SRA) under BioProject PRJNA241291. The whole genome assembly of A. alpina has been deposited at DDBJ/ EMBL/GenBank under the accession JNGA00000000. The version described in this manuscript is version JNGA01000000.

Received 16 September 2014; accepted 10 December 2014; published 2 February 2015

\section{References}

1. The Arabidopsis Genome Initiative. Analysis of the genome sequence of the flowering plant Arabidopsis thaliana. Nature 408, 796-815 (2000).

2. Hu, T. T. et al. The Arabidopsis lyrata genome sequence and the basis of rapid genome size change. Nature Genet. 43, 476-481 (2011).

3. Wang, $X$. et al. The genome of the mesopolyploid crop species Brassica rapa. Nature Genet 43, 1035-1039 (2011). 
4. Dassanayake, M. et al. The genome of the extremophile crucifer Thellungiella parvula. Nature Genet. 43, 913-918 (2011).

5. Wu, H-J. et al. Insights into salt tolerance from the genome of Thellungiella salsuginea. Proc. Natl Acad. Sci. USA 109, 12219-12224 (2012).

6. Yang, R. et al. The reference genome of the halophytic plant Eutrema salsugineum. Front. Plant Sci. 4, 46 (2013).

7. Cheng, S. et al. The Tarenaya hassleriana genome provides insight into reproductive trait and genome evolution of crucifers. Plant Cell 25, 2813-2830 (2013).

8. Haudry, A. et al. An atlas of over 90,000 conserved noncoding sequences provides insight into crucifer regulatory regions. Nature Genet. 45, 891-898 (2013).

9. Slotte, T. et al. The Capsella rubella genome and the genomic consequences of rapid mating system evolution. Nature Genet. 45, 831-835 (2013).

10. Liu, S. et al. The Brassica oleracea genome reveals the asymmetrical evolution of polyploid genomes. Nature Commun. 5, 3930 (2014).

11. Tedder, A., Ansell, S. W., Lao, X., Vogel, J. C. \& Mable, B. K. Sporophytic selfincompatibility genes and mating system variation in Arabis alpina. Ann. Bot. 108, 699-713 (2011).

12. Wingler, A., Stangberg, E. J., Saxena, T. \& Mistry, R. Interactions between temperature and sugars in the regulation of leaf senescence in the perennial herb Arabis alpina L. J. Integr. Plant Biol. 54, 595-605 (2012).

13. Wang, R. et al. PEP1 regulates perennial flowering in Arabis alpina. Nature 459, 423-427 (2009).

14. Sanmiguel, P. \& Bennetzen, J. L. Evidence that a recent increase in maize genome size was caused by the massive amplification of intergene retrotransposons. Ann. Bot. 82, 37-44 (1998).

15. Maumus, F. \& Quesneville, H. Ancestral repeats have shaped epigenome and genome composition for millions of years in Arabidopsis thaliana. Nature Commun. 5, 4104 (2014)

16. Ma, J. \& Bennetzen, J. L. Rapid recent growth and divergence of rice nuclear genomes. Proc. Natl Acad. Sci. USA 101, 12404-12410 (2004).

17. Zhang, X. et al. Genome-wide high-resolution mapping and functional analysis of DNA methylation in arabidopsis. Cell 126, 1189-1201 (2006).

18. Roudier, F. et al. Integrative epigenomic mapping defines four main chromatin states in Arabidopsis. EMBO J. 30, 1928-1938 (2011).

19. Hollister, J. D., Smith, L. M., Guo, Y-L., Ott, F., Weigel, D. \& Gaut, B. S. Transposable elements and small RNAs contribute to gene expression divergence between Arabidopsis thaliana and Arabidopsis lyrata. Proc. Natl Acad. Sci. USA 108, 2322-2327 (2011).

20. Hall, A. E., Kettler, G. C. \& Preuss, D. Dynamic evolution at pericentromeres. Genome Res. 16, 355-364 (2006).

21. Wijnker, E. et al. The genomic landscape of meiotic crossovers and gene conversions in Arabidopsis thaliana. eLife 2, e01426 (2013).

22. Kuittinen, H. et al. Comparing the linkage maps of the close relatives Arabidopsis lyrata and A. thaliana. Genetics 168, 1575-1584 (2004).

23. Zhang, X., Bernatavichute, Y. V., Cokus, S., Pellegrini, M. \& Jacobsen, S. E. Genome-wide analysis of mono-, di- and trimethylation of histone $\mathrm{H} 3$ lysine 4 in Arabidopsis thaliana. Genome Biol. 10, R62 (2009).

24. Cokus, S. J. et al. Shotgun bisulphite sequencing of the Arabidopsis genome reveals DNA methylation patterning. Nature 452, 215-219 (2008).

25. Becker, C. et al. Spontaneous epigenetic variation in the Arabidopsis thaliana methylome. Nature 480, 245-249 (2011).

26. Law, J. A. \& Jacobsen, S. E. Establishing, maintaining and modifying DNA methylation patterns in plants and animals. Nature Rev. Genet. 11, 204-220 (2010)

27. Zemach, A., McDaniel, I. E., Silva, P. \& Zilberman, D. Genome-wide evolutionary analysis of eukaryotic DNA Methylation. Science 328, 916-919 (2010)

28. Matzke, M., Kanno, T., Daxinger, L., Huettel, B. \& Matzke, A. J. M. RNA-mediated chromatin-based silencing in plants. Curr. Opin. Cell Biol. 21, 367-376 (2009).

29. Tsukahara, S., Kobayashi, A., Kawabe, A., Mathieu, O., Miura, A. \& Kakutani, T. Bursts of retrotransposition reproduced in Arabidopsis. Nature 461, 423-426 (2009).
30. Mirouze, M. et al. Selective epigenetic control of retrotransposition in Arabidopsis. Nature 461, 427-430 (2009).

\section{Acknowledgements}

We thank José Jiménez-Gómez for helpful discussion throughout the entire project and Marcus Koch, Maarten Koornneef as well as Miltos Tsiantis for critical reading of the manuscript. We apologize to all colleagues whose work has not been cited here because of space constraints. The TRANSNET consortium was initiated based on the funding of PLANT KBBE 'Transcriptional networks and their evolution in the Brassicaceae (TRANSNET)'. B.S. was supported by a postdoctoral fellowship from the TRANSNET consortium. C.C. was supported by a postdoctoral fellowship first from Investissements d'Avenir ANR-10-LABX-54 MEMO LIFE and then from the European Union EpiGeneSys FP7 Network of Excellence (number 257082). R.C.M.T. was supported by Investissements d'Avenir ANR-10-LABX-54 MEMO LIFE. J.L.M was supported by an Alexander von Humboldt Postdoctoral Fellowship. C.B.S was supported by a postdoctoral fellowship from the TRANSNET consortium. R.I.F. was supported by a post-doctoral Juan de la Cierva contract (JCI-2010-07909) from Ministerio de Ciencia e Innovación (MICINN). A.P. was supported by the research grant PE-1853/2 from German Research Foundation. Work in the Carbonero's group was supported by the Spanish grants BFU2009-11809 and Consolider CSD2007-00057 from Ministerio de Ciencia e Innovación (MICINN). C.A-B. laboratory was funded by grant BIO2013-45407-P from the Ministerio de Economía $y$ Competitividad of Spain. Work in the Colot group was supported by the Agence Nationale de la Recherche (Investissements d'Avenir ANR-10-LABX-54 MEMO LIFE and ANR-11 IDEX-0001-02 PSL* Research University) and the European Union (EpiGeneSys FP7 Network of Excellence number 257082). Work in the Weigel group was supported by an FP7 project AENEAS and the TRANSNET consortium. Work in the Quesneville group was supported by the TRANSNET consortium and by the French national research agency (ANR-08-KBBE-012). Work in the Lysak group was supported by a research grant from the Czech Science Foundation (P501/12/G090) and by the European Social Fund (projects CZ.1.07/2.3.00/30.0037 and CZ.1.07/2.3.00/20.0189). Work on A. alpina in the Coupland laboratory is partly funded by the Cluster of Excellence on Plant Sciences (CEPLAS). Work in the Paz-Ares laboratory has been supported by the French-German-Spanish Trilateral program on Plant Genomics (grant TRANSNET) and Spanish Ministry of Economy competiveness (CONSOLIDER 2007-28317, BIO2011-30546). Schneeberger, Coupland, Weigel and Pecinka groups were supported by the Max Planck Society.

\section{Author contributions}

E.M.W., S.C.S., C.A-B., F.R., P.C., J.P.A., S.J.D., A.P., H.Q., V.C., M.A.L., D.W., G.C. and K.S. conceived this study and supervised experiments and analyses. L.C., M.C.A., B.S., S.B., L.C., J.L.M., M.C.B., N.B., T.P., L.D.L., I.M. and C.B.S. prepared samples for DNA and RNA sequencing. B.S. prepared samples and performed ChIP and MeDIP experiments. C.B. prepared samples and performed bisulphite experiments. N.W., M.C.A. and C.A-B. constructed genetic maps. T.M. and M.A.L. conducted FISH, chromosome painting and karyotype evolution analysis. K.J.V.N., E.M.W. and N.W. conducted de novo assembly of A. alpina. C.K. conducted de novo assembly of A. montbretiana. G.V.J., E.M.W., C.B.S. and M.Z. performed genome annotations of A. alpina together with all participants of the annotation jamboree held in 2012 in Paris, France. E.M.W. performed genome annotations of A. montbretiana. E.M.W., V.R. and C.C. conducted expression analyses. F.M. performed transposon annotations. E.M.W., F.M., M.P. and K.S. performed transposon analysis. E.M.W., C.C., R.C.M.T. and K.S. performed analysis of ChIP-seq and MeDIP-seq data. E.M.W., C.B., J.H. and K.S. performed BS-seq analysis. E.M.W., V.R. and K.S. conducted comparative genomic analyses. E.M.W. and K.S. wrote the paper with contributions from all authors. 